\title{
Estudo transversal com dados radiográficos e documentais de pacientes submetidos a implantes Cone Morse
}

\author{
Cross-sectional study with radiographic and documental data of patients submitted to Cone Morse \\ implants
}

Estudio transversal con datos radiográficos y documentales de pacientes sometidos a implantes de Cono Morse

\section{Resumo}

O objetivo deste trabalho foi realizar um levantamento transversal descritivo com base em exames radiográficos e informações dos prontuários de pacientes submetidos a instalação de implantes Cone Morse Screw Platinum® em uma clínica privada na cidade de Curitiba, PR, Brasil. Foram avaliados os prontuários e os exames radiográficos dos pacientes com idade igual ou superior a 18 anos, de ambos os sexos, atendidos no período de fevereiro de 2015 e junho de 2019. Foram incluídos apenas aqueles com tempo mínimo de instalação de seis meses e que s prontuários estivessem completos. Os dados coletados foram: sexo (masculino ou feminino), idade (em anos), tempo que o implante estava instalado (em meses), arcada (maxila ou mandíbula) e região (anterior ou posterior), posição do implante em relação à altura da crista óssea marginal (infraósseos, em nível ósseo, ou supraósseos). Um total de 27 pacientes (13 homens e 14 mulheres) e 131 implantes foram avaliados. A mediana da idade foi 59 anos e de tempo de instalação do implante foram 15 meses. Quanto à arcada, 70,2\% estavam em maxila e a região posterior teve frequência de $63,3 \%$. Um total de 88 implantes estava em posição infraóssea. Pode-se concluir que a maioria dos implantes avaliados estava, em relação à crista óssea marginal, em posição infraóssea, que é considerada a ideal. A maxila, tanto em região anterior como posterior foi a mais reabilitada, o que pode indicar a importância da questão estética por parte dos pacientes aqui analisados.

Palavras-chave: Implantes dentários; Interpretação de imagem radiográfica; Documentos.

\section{Abstract}

The aim of this study was to carry out a descriptive cross-sectional survey based on radiographic examinations and information from medical records of patients who underwent installation of Cone Morse Screw Platinum ${ }^{\circledR}$ implants in a private clinic in the city of Curitiba, PR, Brazil. The medical records and radiographic examinations of patients aged 18 years or over, of both genders, treated in the period between February 2015 and June 2019 were included. Only those with a minimum installation time of six months and who were s medical records were complete. The data 
collected were: gender (male or female), age (in years), time the implant was installed (in months), arch (maxilla or mandible) and region (anterior or posterior), implant position in relation to height of the marginal bone crest (infraosseous, at the bone level, or supraosseous). A total of 27 patients (13 men and 14 women) and 131 implants were evaluated. The median age was 59 years and implant placement time was 15 months. As for the arch, $70.2 \%$ were in the maxilla and the posterior region had a frequency of 63.3\%. A total of 88 implants were in the infraosseous position. It can be concluded that most of the evaluated implants were, in relation to the marginal bone crest, in an infraosseous position, which is considered the ideal. The maxilla, both anteriorly and posteriorly, was the most rehabilitated, which may indicate the importance of the aesthetic issue for the patients analyzed here.

Keywords: Dental implants; Radiographic image interpretation; Records.

\section{Resumen}

El objetivo de este estudio fue realizar una encuesta descriptiva transversal basada en exámenes radiográficos e información de historias clínicas de pacientes que fueron sometidos a la instalación de implantes Cone Morse Screw Platinum ${ }^{\circledR}$ en una clínica privada de la ciudad de Curitiba, PR, Brasil. Se incluyeron las historias clínicas y exámenes radiográficos de los pacientes de 18 años o más, de ambos sexos, atendidos en el período comprendido entre febrero de 2015 y junio de 2019. Solo aquellos con un tiempo mínimo de instalación de seis meses y que tenían una historia clínica completa. Los datos recogidos fueron: sexo (hombre o mujer), edad (en años), tiempo de instalación del implante (en meses), arco (maxilar o mandíbula) y región (anterior o posterior), posición del implante en relación con la altura del cresta ósea marginal (infraósea, a nivel óseo o supraósea). Se evaluaron un total de 27 pacientes (13 hombres y 14 mujeres) y 131 implantes. La mediana de edad fue de 59 años y el tiempo de colocación del implante fue de 15 meses. En cuanto al arco, el 70,2\% estaban en el maxilar y la región posterior tenía una frecuencia del 63,3\%. Un total de 88 implantes estaban en posición infraósea. Se puede concluir que la mayoría de los implantes evaluados se encontraban, en relación con la cresta ósea marginal, en una posición infraósea, que se considera la ideal. El maxilar, tanto anterior como posterior, fue el más rehabilitado, lo que puede indicar la importancia del tema estético para los pacientes aquí analizados.

Palabras clave: Implantes dentales; Interpretación de imagen radiográfica; Documento.

\section{Introdução}

Os implantes dentários são uma opção de tratamento para reabilitação dentária, com eficácia comprovada há muito tempo, de pacientes desdentados total ou parcialmente (Branemark et al., 1977). A prática da Implantodontia vem se dissipando na comunidade odontológica de forma intensa, e a utilização de implantes dentários tem se tornado cada vez mais viável para a maioria dos casos (Clark \& Levin, 2019; Silva et al., 2020). No entanto, os implantes precisam ser instalados com posicionamento o mais adequado possível, a fim de serem obtidos resultados em termos de longevidade. Logo, a reabilitação da perda dentária com implantes demanda um planejamento pré-operatório completo (cirúrgico e protético), como um requisito fundamental para o sucesso do resultado reabilitador (Hultin et al., 2012).

Os exames de imagem podem oferecer ao operador informações acerca das condições anatômicas que são fundamentais ao planejamento mais adequado, tanto para a instalação cirúrgica quanto para a reabilitação protética. Assim, pensando na avaliação de implantes instalados, os exames de imagem podem auxiliar na avaliação do tratamento executado (Merheb et al., 2015). Um importante parâmetro a ser considerado nesses exames se trata da crista óssea marginal (Merheb et al., 2015; Silva et al., 2020). Em trabalho recente, Linkevicius et al. (2020) demonstraram uma avaliação de implantes dentários relacionando os níveis da crista óssea ao redor de implantes, em medições a partir de exames radiográficos, método adotado na presente pesquisa.

Um ponto que parece influenciar a manutenção da crista óssea marginal é o desenho do implante. O desenho conhecido como Cone Morse pode ser indicado para solucionar diversos casos que envolvam desde próteses unitárias a múltiplas, sobredentadura e protocolo (Valente et al., 2015; Veríssimo et al., 2021). O estudo das relações entre superfícies justapostas cônicas, preconizado por Stephen A. Morse, na década de 1960, vem sendo utilizado em diversas áreas. No entanto, esse tipo de estudo com aplicação clínica foi inserido no mercado odontológico apenas três décadas depois, nos anos 1090 (Kfouri et al., 2013).

Em 2014, Schmit et al. já observaram vantagens e manutenção da crista óssea marginal quando do uso do desenho 
Cone Morse. Sob cargas, demonstraram menor aumento de microfendas entre os componentes e a plataforma do implante, menor perda de torque, compensação de altas tensões e proteção do parafuso da prótese no caso de sobrecargas. Além disso, outro ponto importante do desenho dos implantes é a plataforma switch, descrita como conexão protética de menor diâmetro do que o corpo do implante, na região de conexão. Essa variação melhora a distribuição do estresse e diminui a perda da crista óssea marginal (Pita et al., 2011; Mangano et al., 2011; 2015). Dados confirmados em outro estudo clínico (Tang et al., 2017) também demonstraram que implantes que combinam a conexão do Cone Morse com a plataforma switch podem reduzir a perda da crista óssea marginal, incluindo maior estabilidade primária (Valente et al., 2015).

Casseta et al. (2016) concordaram que o trauma oclusal ou o excesso de carga podem levar à perda óssea da crista marginal ao redor de implantes dentários. Ademais, características como desenho oclusal das coroas sobre implante, qualidade e volume do osso, hábitos e dieta, presença de parafunção (bruxismo) também podem influenciar a altura da crista óssea marginal. Castro et al. (2014) demonstraram que a posição infraóssea do implante Cone Morse favorece também sua longevidade em boca.

Em 2010, Palinkas et al. já evidenciaram a relação entre as forças mastigatórias, idade e sexo, o que pode, naturalmente, influenciar as reabilitações com implantes dentários, especialmente porque esses não possuem o ligamento periodontal, presente nos dentes naturais.

Ainda, relacionando a condição dos implantes dentários com as cargas mastigatórias, de acordo com Flanagan (2017), a região posterior também pode desempenhar uma intensidade até três vezes maior do que a região anterior. Por isto, pode ser importante considerar questões de desenho do implante e das coroas nesta região.

Assim, a partir de dados radiográficos e demográficos, tanto profissionais como fabricantes de implantes podem sedimentar estratégias mais favoráveis no sentido de preservar ao máximo os tratamentos, resultando numa melhor perspectiva de longevidade dos mesmos. Neste contexto, o objetivo deste trabalho foi realizar um estudo transversal descritivo, qualitativo de implantes Cone Morse Screw Platinum ${ }^{\circledR}$ instalados em uma clínica especializada de prática privada, a partir da avaliação de exames radiográficos e de dados demográficos dos pacientes.

\section{Metodologia}

Este trabalho foi submetido ao Comitê de Ética em Pesquisa da Universidade Positivo (registro n. ${ }^{\circ}$ 4.025.968). O desenho de estudo adotado foi transversal, com amostra de conveniência, de base documental. O método aqui descrito foi adaptado de Merheb (2015).

Os dados foram coletados com base nos registros dos prontuários do Instituto Odontológico Kopp, localizado em Curitiba, PR, Brasil, cujos pacientes receberam implantes Cone Morse Screw Platinum ${ }^{\circledR}$ (Kopp Sistema de Implante Dental, Curitiba, PR, Brasil). Os dados demográficos coletados foram: sexo (masculino ou feminino), idade (em anos), tempo que o implante está instalado (conforme data da última radiografia panorâmica), arcada (maxila ou mandíbula) e região (anterior ou posterior). Além disso, a altura mesio-distal do osso ao lado dos implantes foi estabelecida em todas as radiografias e classificada em: implantes infraósseos, nos quais a plataforma do implante estava abaixo da crista óssea marginal; implantes em nível ósseo, nos quais a plataforma do implante estava nivelada com a crista óssea marginal; ou implantes supraósseos, nos quais a plataforma do implante estava acima da crista óssea marginal. Todas as imagens radiográficas foram analisadas primeiramente por apenas um examinador e em seguida foram conferidas por outro examinador conspirado padrão-ouro, com 20 anos de experiência em Implantodontia.

Foram incluídos dados de pacientes de ambos os sexos, com 18 anos de idade ou mais, de qualquer raça, que tivessem recebido os implantes entre fevereiro de 2015 e junho de 2019, devidamente registrados nos prontuários, e que possuíam radiografias panorâmicas com no mínimo seis meses da instalação dos implantes. 
Os dados coletados foram tabulados e analisados quanto às frequências absoluta e relativa, em SPSS, versão 23.0 (IBM SPSS Statistics for Windows, Version 23.0. Armonk, Nova York, Estados Unidos da América).

\section{Resultados}

Foram coletados dados dos prontuários de 27 pacientes (13 homens e 14 mulheres), que receberam um total de 131 implantes. Conforme foi possível observar, o número de implantes em homens $(\mathrm{n}=74)$ foi superior às mulheres $(\mathrm{n}=57)$. A mediana da idade foi de 59 anos (mínimo 27 e máximo de 76), e do tempo de instalação dos implantes foi de 15 meses (mínimo seis e máximo de 55) (Tabela 1).

Tabela 1 - Distribuição do número de implantes de acordo com os dados demográficos dos prontuários de pacientes incluídos no estudo $(\mathrm{n}=27)$.

\begin{tabular}{ll}
\hline Variável & $\mathbf{n}(\boldsymbol{\%})$ \\
\hline Sexo & $74(56,5)$ \\
Masculino & $57(43,5)$ \\
Feminino & Mediana (mín - máx) \\
& $59(27-76)$ \\
Idade (em anos) & $15(6-55)$ \\
Tempo que implante estava instalado (em meses) & $\mathbf{n}(\%)$ \\
Implantes por arcada e região & $36(27,5)$ \\
Maxila anterior & $56(42,7)$ \\
Maxila posterior & $12(9,2)$ \\
Mandíbula anterior & $27(20,6)$ \\
Mandíbula posterior & \\
\hline
\end{tabular}

Fonte: Autores.

Também se pode destacar que um número maior de implantes foi instalado em região de maxila ( $\mathrm{n}=92 ; 70,2 \%)$, e 39 foram em mandíbula $(29,8 \%)$, sendo a maioria ocorreu em região posterior, tanto em maxila quanto em mandíbula $(\mathrm{n}=83$, $63,3 \%$ ) (Tabela 1).

A Tabela 2 apresenta a distribuição da classificação da posição dos implantes conforme as demais variáveis de interesse. Implantes infraósseos foram os mais prevalentes $(n=88)$, com distribuição igual entre idade maior ou igual ou menor do que 59 anos. Mesmo entre os tempos maiores ou menores do que 15 meses, a posição infraóssea também permaneceu prevalente; um número maior de implantes em condição infraóssea também foi encontrado em maxila (53,5\%). 
Tabela 2 - Classificação radiográfica da posição do implante em relação à altura da crista óssea marginal conforme as variáveis demográficas dos pacientes incluídos no estudo $(\mathrm{n}=27)$.

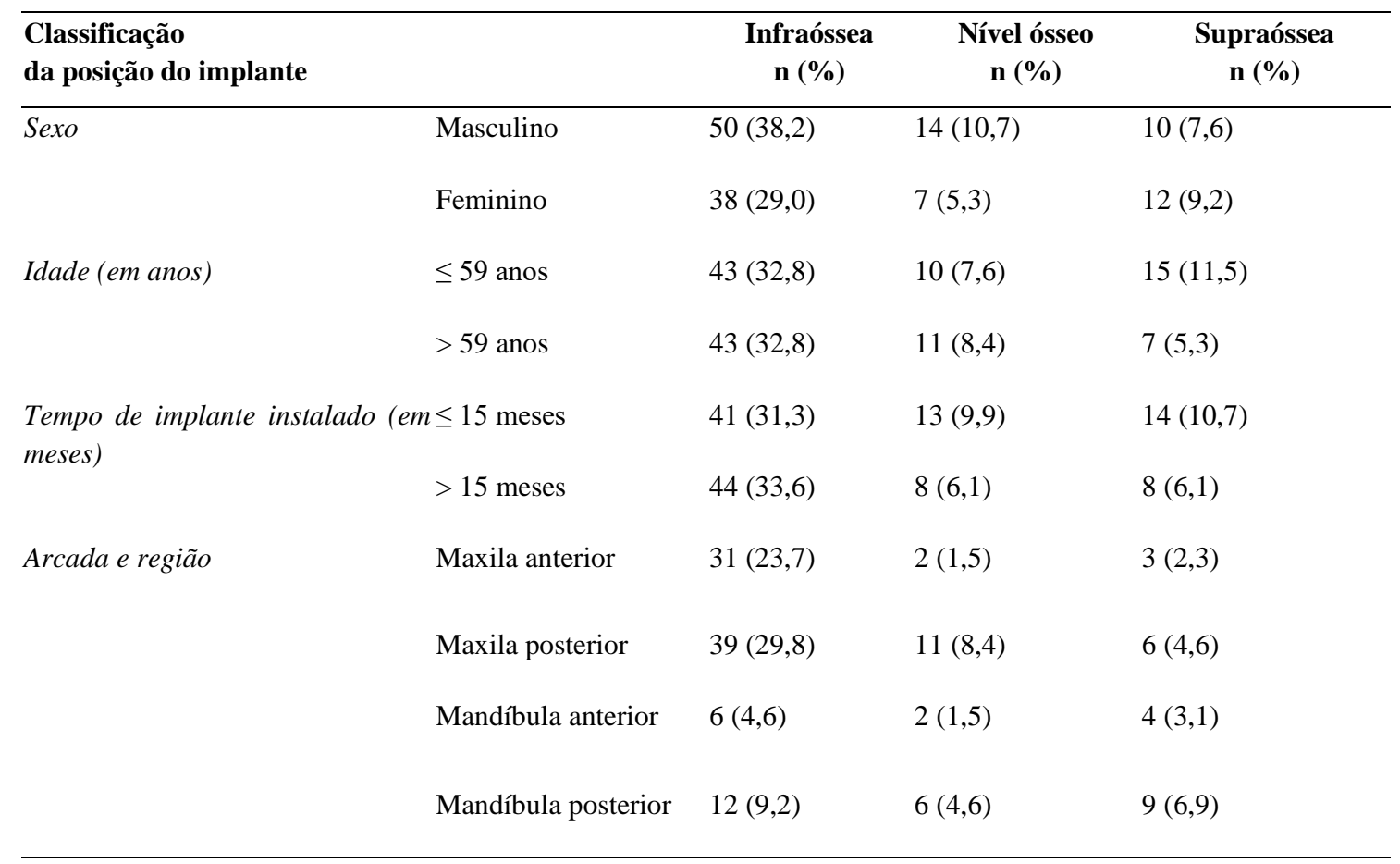

Fonte: Autores.

Somando-se os implantes em homens e mulheres, mais de 50\% dos implantes foram encontrados em maxila e permanecendo infraósseos. Essa condição infraóssea também foi prevalente ao serem somados os percentuais dos implantes maxilares dos pacientes de todas as idades do estudo (52\%), independentemente do tempo em boca de acordo com os exames $(52,8 \%)$ (Tabela 3$)$.

Quanto à distribuição anterior ou posterior, os implantes maxilares ocorreram mais em região posterior (30,3\%). Já na mandíbula os totais foram menores, mas também com menor variação entre as diferentes alturas de crista óssea marginal. Nos homens 10 implantes mandibulares estavam infraósseos e seis estavam supraósseos; nas mulheres o padrão foi semelhante, com oito implantes mandibulares infraósseos e sete supraósseos.

Quanto à idade, o mesmo número de implantes infraósseos e supraósseos foi encontrado $(n=9)$, em pacientes com menos de 59 anos. Em radiografias com tempo de implante inferior a 15 meses os implantes mandibulares supraósseos $(n=7)$ foram mais prevalentes que os infraósseos $(n=5)$, sendo que em tempos superiores 15 meses, o número absoluto de implantes mandibulares supraósseos foi de oito e os infraósseos foram seis. Ainda, tanto na região anterior quanto na posterior, os implantes infraósseos ( $n=6$ e $n=12$, respectivamente) foram superiores aos supraósseos ( $n=4$ e n $=9$, respectivamente). 
Tabela 3 - Associação entre a altura da crista marginal e a plataforma do implante, por arcada, conforme demais variáveis dos pacientes incluídos no estudo $(\mathrm{n}=27)$.

\begin{tabular}{|c|c|c|c|c|}
\hline \multicolumn{5}{|c|}{ MAXILA } \\
\hline Classificação da altura da crista marginal & & $\begin{array}{c}\text { Infraóssea } \\
\text { n (\%) }\end{array}$ & $\begin{array}{c}\text { Nível ósseo } \\
\text { n }(\%)\end{array}$ & $\begin{array}{c}\text { Supraóssea } \\
\text { n }(\%)\end{array}$ \\
\hline \multirow[t]{2}{*}{ Sexo } & Masculino & $40(30,5)$ & $11(8,4)$ & $4(3,1)$ \\
\hline & Feminino & $30(22,9)$ & $2(1,5)$ & $5(3,8)$ \\
\hline \multirow[t]{2}{*}{ Idade (em anos) } & $\leq 59$ anos & $34(26,0)$ & $8(6,1)$ & $6(4,6)$ \\
\hline & $>59$ anos & $34(26,0)$ & $5(3,8)$ & $3(2,3)$ \\
\hline \multirow[t]{2}{*}{ Tempo de implante instalado (em meses) } & $\leq 15$ meses & $36(27,6)$ & $11(8,4)$ & $7(5,2)$ \\
\hline & $>15$ meses & $33(25,2)$ & $2(1,5)$ & $2(1,5)$ \\
\hline \multirow[t]{2}{*}{ Região } & Anterior & $31(23,6)$ & $2(1,5)$ & $3(2,3)$ \\
\hline & Posterior & $39(30,3)$ & $11(8,4)$ & $6(4,6)$ \\
\hline \multicolumn{5}{|c|}{ MANDÍBULA } \\
\hline Classificação da altura da crista marginal & & $\begin{array}{c}\text { Infraóssea } \\
\text { n (\%) }\end{array}$ & $\begin{array}{c}\text { Nível ósseo } \\
\text { n (\%) }\end{array}$ & $\begin{array}{c}\text { Supraóssea } \\
\text { n }(\%)\end{array}$ \\
\hline \multirow[t]{2}{*}{ Sexo } & Masculino & $10(7,6)$ & $3(2,3)$ & $6(4,6)$ \\
\hline & Feminino & $8(6,1)$ & $5(3,9)$ & $7(5,4)$ \\
\hline \multirow[t]{2}{*}{ Idade (em anos) } & $\leq 59$ anos & $9(6,9)$ & $2(1,6)$ & $9(6,9)$ \\
\hline & $>59$ anos & $9(6,9)$ & $6(4,6)$ & $4(3,1)$ \\
\hline \multirow[t]{2}{*}{ Tempo de implante instalado (em meses) } & $\leq 15$ meses & $5(3,9)$ & $2(1,5)$ & $7(5,4)$ \\
\hline & $>15$ meses & $8(6,2)$ & $6(4,6)$ & $6(4,6)$ \\
\hline \multirow[t]{2}{*}{ Região } & Anterior & $6(4,6)$ & $2(1,5)$ & $4(3,1)$ \\
\hline & Posterior & $12(9,1)$ & $6(4,6)$ & $9(6,9)$ \\
\hline
\end{tabular}

Fonte: Autores.

\section{Discussão}

No intuito de serem obtidos dados iniciais e nortear estudos mais aprofundados, estudos descritivos como o que aqui se apresenta podem apontar para alguma direção futura, levantando questões de relevância voltadas para a melhoria da qualidade da aplicação clínica dos implantes, tais como: levantamento minucioso de características biológicas dos pacientes, padronização na instalação cirúrgica, acompanhamento clínico sistemático dos tratamentos e avaliações padronizadas de imagem, dados já apontados por Klinge et al. (2018).

No que diz respeito à escolha do desenho do implante, sabe-se que a relação entre as superfícies de contato tecido ósseo-implante-componente protético pode influenciar o comportamento biológico dos implantes (Castro et al., 2014). Essas características podem ser consideradas durante a elaboração dos planejamentos reabilitadores, a fim de propiciar ao paciente (e ao fabricante) a melhor abordagem técnica e clínica, considerando em última análise uma reabilitação funcional e estética, com previsibilidade de manutenção (Bolle et al., 2016).

Enquanto que para Casseta et al. (2016), uma importante condição para a manutenção da crista óssea marginal é a plataforma switch, para Romanos E Javed (2014), a perda da crista óssea marginal também está relacionada com outros fatores como a micromovimentação do componente protético, o posicionamento tridimensional do implante e a espessura da crista. 
De acordo com Pessoa et al. (2017) o implante Cone Morse deve diminuir a perda da crista óssea marginal, especialmente quando se encontra na posição infraóssea (Novaes et al., 2009; Degidi et al., 2011; Bolle, et al., 2016). Ao longo do tempo, observa-se preservação da crista óssea e perda pequena ao redor dos implantes (Degidi et al., 2011; Degidi $e t$ al., 2016). Por isso, Koutouzis et al. (2014) indicam que esses implantes sejam instalados a $2 \mathrm{~mm}$ infraósseos. No atual trabalho, a informação da instalação cirúrgica dos implantes não foi relatada, mas segundo o próprio fabricante, o implante utilizado também deve seguir este parâmetro (Kopp, 2011).

Sobre as características demográficas encontradas no presente trabalho, os pacientes podem ter buscado o tratamento com implantes dentários por motivos estéticos, uma vez que a maioria dos implantes foi instalada em maxila anterior e posterior. Assim, esse tipo de dado deve fazer parte do prontuário dos pacientes, ou seja, a motivação pela busca de implantes dentários. Korfage et al. (2018) descrevem que muitos pacientes buscam o tratamento com implantes erroneamente pensando que a exigência será por cuidados mínimos e que o procedimento tem durabilidade infinita. Daí, também, a importância de se informar/educar adequadamente o paciente para que o tratamento tenha real longevidade.

Quanto ao número de implantes, de modo geral pode-se dizer que o presente trabalho apresentou uma amostra de quase cinco implantes por paciente, ou seja, o tratamento com reabilitação de áreas edêntulas múltiplas foi identificado, fato semelhante ao relatado por Mangano et al. (2011) e Mericske-Stern E Worni (2014).

Com relação à idade, a mediana foi de 59 anos, corrobora que do ponto de vista biológico, sabe-se que com o aumento da idade há um incremento das perdas dentárias (Howe et al., 2019).

No atual trabalho, a maior parte dos implantes $(67,2 \%)$ se encontrava em condição infraóssea, e em maior número na maxila. No entanto, ao serem observados os implantes mandibulares, notou-se uma pequena diferença entre o número de implantes mandibulares infraósseos e os supraósseos (condição desfavorável). Implantes supraósseos podem ter um prognóstico ruim, especialmente em região estética; a exposição de roscas, por exemplo, pode levar ao acúmulo de biofilme e atração de microrganismos, fator negativo para a manutenção da crista óssea marginal (Hudieb et al., 2019).

Quanto às limitações desta pesquisa, destaca-se o desenho de estudo (transversal), o qual não permite que relações causais sejam estabelecidas (Belbasis \& Belou, 2018). Também há outros dados que seriam relevantes para pesquisas desse caráter, mas que não foram coletadas, como os hábitos do paciente (higiene, fumo, dentre outros).

Por fim, para Clark e Levin (2019) afirma que, independentemente da modalidade de tratamento com implantes dentários, as características mais críticas para o sucesso com os implantes são: manutenção permanente, implementação de protocolos rigorosos de acompanhamento e um planejamento do tratamento com responsabilidade ética e baseado em evidências.

\section{Conclusão}

A amostra analisada apresentou a maioria dos implantes em posição infraóssea e em maxila, o que reflete, primeiramente uma condição clínica adequada e sem segundo lugar, que os aspectos estéticos podem ter sido os motivadores para a busca de reabilitação por parte dos pacientes avaliados. Apesar das limitações deste estudo, o mesmo pode servir como base para trabalhos futuros, confirmando a necessidade de protocolos clínicos mais rigorosos nas reabilitações bucais com implantes dentários.

\section{Referências}

Brånemark, P. I., Hansson, B. O., Adell, R., Breine, U., Lindström, J., Hallén, O., \& Ohman, A. (1977). Osseointegrated implants in the treatment of the edentulous jaw. Experience from a 10-year period. Scandinavian Journal of Plastic and Reconstructive Surgery. Supplementum, 16, 1-132.

Belbasis, L., \& Bellou, V. (2018). Introduction to epidemiological Studies. Methods in Molecular Biology (Clifton, N.J.), 1793, 1-6. https://doi.org/10.1007/978-1-4939-7868-7_1 
Bolle, C., Gustin, M. P., Fau, D., Boivin, G., Exbrayat, P., \& Grosgogeat, B. (2016). Soft tissue and marginal bone adaptation on platform-switched implants with a morse cone connection: a histomorphometric study in dogs. The International Journal of Periodontics \& Restorative Dentistry, 36(2), 221-228. https://doi.org/10.11607/prd.2254

Cassetta, M., Di Mambro, A., Giansanti, M., \& Brandetti, G. (2016). The survival of Morse Cone-connection implants with platform switch. The International Journal of Oral \& Maxillofacial Implants, 31(5), 1031-1039. https://doi.org/10.11607/jomi.4225

Castro, D. S., Araujo, M. A., Benfatti, C. A., Araujo, C., Piattelli, A., Perrotti, V., \& Iezzi, G. (2014). Comparative histological and histomorphometrical evaluation of marginal bone resorption around external hexagon and Morse cone implants: an experimental study in dogs. Implant Dentistry, 23(3), 270-276. https://doi.org/10.1097/ID.0000000000000089

Clark, D., \& Levin, L. (2019). In the dental implant era, why do we still bother saving teeth? Dental Traumatology, 35(6), 368-375. https://doi.org/10.1111/edt.12492

Degidi, M., Perrotti, V., Shibli, J. A., Novaes, A. B., Piattelli, A., \& Iezzi, G. (2011). Equicrestal and subcrestal dental implants: a histologic and histomorphometric evaluation of nine retrieved human implants. Journal of Periodontology, 82(5), 708-715. https://doi.org/10.1902/jop.2010.100450

Degidi, M., Daprile, G., \& Piattelli, A. (2017). Marginal bone loss around implants with platform-switched Morse-cone connection: a radiographic crosssectional study. Clinical Oral Implants Research, 28(9), 1108-1112. https://doi.org/10.1111/clr.12924

Flanagan D. (2017). Bite force and dental implant treatment: a short review. Medical Devices, 10, 141-148. https://doi.org/10.2147/MDER.S130314

Howe, M. S., Keys, W., \& Richards, D. (2019). Long-term (10-year) dental implant survival: A systematic review and sensitivity meta-analysis. Journal of Dentistry, 84, 9-21. https://doi.org/10.1016/j.jdent.2019.03.008

Hudieb, M. I., Wakabayashi, N., Abu-Hammad, O. A., \& Kasugai, S. (2019). Biomechanical effect of an exposed dental implant's first thread: a threedimensional finite element analysis study. Medical Science Monitor: International Medical Journal of Experimental and Clinical Research, 25, 3933-3940. https://doi.org/10.12659/MSM.913186

Hultin, M., Svensson, K. G., \& Trulsson, M. (2012). Clinical advantages of computer-guided implant placement: a systematic review. Clinical Oral Implants Research, 23(Sup 6), 124-135. https://doi.org/10.1111/j.1600-0501.2012.02545.x

Kfouri, F. A. (2013). Versatilidade clínica de componentes protéticos cone morse. Revista Eletrônica da Faculdade de Odontologia da FMU, 2 (2), 1-4.

Klinge, B., Lundström, M., Rosén, M., Bertl, K., Klinge, A., \& Stavropoulos, A. (2018). Dental implant quality register-a possible tool to further improve implant treatment and outcome. Clinical Oral Implants Research, 29 (18), 145-151. https://doi.org/10.1111/clr.13268

Korfage, A., Raghoebar, G. M., Meijer, H., \& Vissink, A. (2018). Patients' expectations of oral implants: a systematic review. European Journal of Oral Implantology, 11 (1), S65-S76.

Koutouzis, T., Neiva, R., Nair, M., Nonhoff, J., \& Lundgren, T. (2014). Cone beam computed tomographic evaluation of implants with platform-switched Morse taper connection with the implant-abutment interface at different levels in relation to the alveolar crest. The International Journal of Oral \& Maxillofacial Implants, 29(5), 1157-1163. https://doi.org/10.11607/jomi.3411

Kopp, G. (2011). Manual cirúrgico Kopp implantes. https://issuu.com/implantkopp/docs/04-manual-cirurgico. Acessado em: 06/2021.

Linkevicius, T., Puisys, A., Linkevicius, R., Alkimavicius, J., Gineviciute, E., \& Linkeviciene, L. (2020). The influence of submerged healing abutment or subcrestal implant placement on soft tissue thickness and crestal bone stability. A 2-year randomized clinical trial. Clinical Implant Dentistry and Related Research, 22(4), 497-506. https://doi.org/10.1111/cid.12903

Mangano, C., Mangano, F., Shibli, J. A., Tettamanti, L., Figliuzzi, M., d'Avila, S., Sammons, R. L., \& Piattelli, A. (2011). Prospective evaluation of 2,549 morse taper connection implants: 1- to 6-year data. Journal of Periodontology, 82(1), 52-61. https://doi.org/10.1902/jop.2010.100243

Mangano, C., Iaculli, F., Piattelli, A., \& Mangano, F. (2015). Fixed restorations supported by morse-taper connection implants: a retrospective clinical study with 10-20 years of follow-up. Clinical Oral Implants Research, 26(10), 1229-1236. https://doi.org/10.1111/clr.12439

Mericske-Stern, R., \& Worni, A. (2014). Optimal number of oral implants for fixed reconstructions: a review of the literature. European Journal of Oral Implantology, 7(2), S133-S153.

Merheb, J., Graham, J., Coucke, W., Roberts, M., Quirynen, M., Jacobs, R., \& Devlin, H. (2015). Prediction of implant loss and marginal bone loss by analysis of dental panoramic radiographs. The International Journal of Oral \& Maxillofacial Implants, 30(2), 372-377. https://doi.org/10.11607/jomi.3604

Novaes, A. B., Jr, Barros, R. R., Muglia, V. A., \& Borges, G. J. (2009). Influence of interimplant distances and placement depth on papilla formation and crestal resorption: a clinical and radiographic study in dogs. The Journal of Oral Implantology, 35(1), 18-27. https://doi.org/10.1563/1548-1336-35.1.18

Palinkas, M., Nassar, M. S., Cecílio, F. A., Siéssere, S., Semprini, M., Machado-de-Sousa, J. P., Hallak, J. E., \& Regalo, S. C. (2010). Age and gender influence on maximal bite force and masticatory muscles thickness. Archives of Oral Biology, 55(10), 797-802. https://doi.org/10.1016/j.archoralbio.2010.06.016

Pessoa, R. S., Sousa, R. M., Pereira, L. M., Neves, F. D., Bezerra, F. J., Jaecques, S. V., Sloten, J. V., Quirynen, M., Teughels, W., \& Spin-Neto, R. (2017). Bone remodeling around implants with external hexagon and morse-taper connections: a randomized, controlled, split-mouth, clinical trial. Clinical Implant Dentistry and Related Research, 19(1), 97-110. https://doi.org/10.1111/cid.12437

Pita, M. S., Anchieta, R. B., Barão, V. A., Garcia, I. R., Jr, Pedrazzi, V., \& Assunção, W. G. (2011). Prosthetic platforms in implant dentistry. The Journal of Craniofacial Surgery, 22(6), 2327-2331. https://doi.org/10.1097/SCS.0b013e318232a706 
Research, Society and Development, v. 11, n. 2, e18811224123, 2022

(CC BY 4.0) | ISSN 2525-3409 | DOI: http://dx.doi.org/10.33448/rsd-v11i2.24123

Romanos, G. E., \& Javed, F. (2014). Platform switching minimises crestal bone loss around dental implants: truth or myth?. Journal of Oral Rehabilitation, 41(9), 700-708. https://doi.org/10.1111/joor.12189

Schmitt, C. M., Nogueira-Filho, G., Tenenbaum, H. C., Lai, J. Y., Brito, C., Döring, H., \& Nonhoff, J. (2014). Performance of conical abutment (Morse Taper) connection implants: a systematic review. Journal of Biomedical Materials Research. Part A, 102(2), 552-574. https://doi.org/10.1002/jbm.a.34709

Silva, R. M. M. da, Rolim, A. K. A., Delgado, L. A., Sousa, J. T., Ribeiro, R. A., Rodrigues, R. de Q. F., \& Rodrigues, R. A. (2020). Cone morse x external hexagon, advantages and disadvantages in the clinical aspect: literature review. Research, Society and Development, 9(7), e454973947. https://doi.org/10.33448/rsd-v9i7.3947

Tang, C. L., Zhao, S. K., \& Huang, C. (2017). Features and advances of Morse taper connection in oral implant. Chinese Journal of Stomatology, 52(1), 5962. https://doi.org/10.3760/cma.j.issn.1002-0098.2017.01.014

Valente, M. L., de C., D. T., Shimano, A. C., Lepri, C. P., \& dos Reis, A. C. (2015). Analysis of the influence of implant shape on primary stability using the correlation of multiple methods. Clinical Oral Investigations, 19(8), 1861-1866. https://doi.org/10.1007/s00784-015-1417-4

Veríssimo, A. H., Souza, J. A. N. de., Oliveira, T. A. de., Gonçalves, A. G., Afonso, F. A. C., \& Souza Júnior, F. A. (2021). Oral rehabilitation with dental implant and immediate loading by guided surgery: case report. Research, Society and Development, 10(1), e4810110854. https://doi.org/10.33448/rsdv10i1.10854 